\title{
Spectral Analysis of Polysomnography in Narcolepsy
}

\author{
Seok Ho Yun ${ }^{1}$, Ho Dong $\mathrm{Choi}^{2}$, and Wan Seok Seo ${ }^{\circledR}$ \\ 'Department of Neuropsychiatry, Yeungnam University, Daegu, Republic of Korea \\ ${ }^{2}$ Department of Psychiatry, Motherth Hospital, Ulsan, Republic of Korea
}

\begin{abstract}
Objective This study was conducted to identify differences between people with narcolepsy and the normal control of delta and theta activity using electroencephalogram (EEG) spectrum analysis of nocturnal polysomnography (PSG).

Methods Seven narcolepsy patients and seven age-sex matched normal controls underwent PSG and multiple sleep latency tests. Participants' non-rapid eye movement (NREM) sleep EEGs in PSG was analyzed using a Fast Fourier Transform technique.

Results While NREM delta activity of people with narcolepsy declined during the first three periods of NREM, there was no change during the 4th period of NREM. The increase in NREM theta activity also lasted until the 3rd period of NREM but did not occur during the 4th period of NREM. In comparing sleep parameters, REM sleep latency in the narcolepsy group was significantly shorter than in controls.

Conclusion These results suggest that people with narcolepsy are likely to have a delta and theta activity-related sleep disturbance mechanism in NREM sleep.

Psychiatry Investig 2017;14(2):193-197
\end{abstract}

Key Words Narcolepsy, Delta activity, Theta activity, Electroencephalogram, Spectral analysis.

\section{INTRODUCTION}

Narcolepsy is a chronic neurological sleep disorder of rapid eye movement (REM) sleep, non-REM (NREM) sleep, and of the sleep-wake cycle, characterized by excessive daytime sleepiness, sudden loss of muscle tone (cataplexy), sleep paralysis, hypnagogic hallucinations, and disturbed night sleep. ${ }^{1,2}$ Narcolepsy is diagnosed by clinical history taking, polysomnography (PSG), multiple sleep latency test (MSLT), and hypocretin (Hcrt) levels in the cerebrospinal fluid. ${ }^{3}$ The international classification of sleep disorders, second edition (ICSDII) provides the following diagnostic criteria for narcolepsy: sleep latency of less than 8 minutes, two or more sleep-onset REM periods (SOREMPs) seen on MSLT following sufficient nocturnal sleep (minimum 6 hours) during the night prior to testing. ${ }^{3}$ PSG not only makes it possible to identify the macroscopic structures of sleep such as sleep stages and SOREMPs, but also enables behavior and movement to be

Received: January 19, 2016 Revised: May 7, 2016

Accepted: July 1, 2016 Available online: October 19, 2016

$\triangle$ Correspondence: Wan Seok Seo, MD, PhD

Department of Neuropsychiatry, College of Medicine, Yeungnam University, 170 Hyeonchung-ro, Daegu 42415, Republic of Korea

Tel: +82-53-620-3344, Fax: +82-53-629-0256, E-mail: sws3901@ynu.ac.kr

(a) This is an Open Access article distributed under the terms of the Creative Commons Attribution Non-Commercial License (http://creativecommons.org/licenses/bync/4.0) which permits unrestricted non-commercial use, distribution, and reproduction in any medium, provided the original work is properly cited. monitored during sleep. However, PSG alone cannot provide detailed pathophysiological information about specific sleep

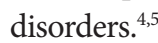

Previous PSG studies on narcoleptics mainly focused on NREM and REM sleep abnormalities such as reduced REM and NREM sleep latency, increased micro-arousal during sleep, increased sleep-wake sleep transition, increased wake time after sleep onset (WASO), and an increased NREM 1st stage. ${ }^{6-8}$ Recent studies have focused on pathophysiology, new diagnostic tools, treatments, and relationships with other sleep disorders using sleep electroencephalogram (EEG) spectral analysis. ${ }^{9-12}$ Spectral analysis of EEG has also been used to evaluate detailed quantitative and temporal changes in EEG frequency bands. ${ }^{13}$

EEG spectral analysis of PSG provides a means of quantifying certain wave shapes over time by applying Fourier transformation to brain wave signals. ${ }^{14}$ The technique might be particularly useful for identifying the functional abnormalities of narcolepsy because it provides information about the distributions and strengths of certain brain wave shapes during sleep. ${ }^{4}$ Mukai et al. ${ }^{15}$ analyzed PSGs of eight drug-naïve narcoleptic patients and eight age-matched controls and found that while normal controls showed a significant decline in delta power during night sleep, narcoleptic patients did not. Studies on the spectral analysis of sleep EEGs have focused mainly on slow wave activity (SWA) to date. SWA has a frequency similar to those of delta activity and prominently appears in 
slow wave sleep (SWS). SWA increases after prolonged prior wakefulness and decreases after sleep onset, that is, SWA has been reported to be positively correlated with human sleep needs. ${ }^{16}$

Narcolepsy has been reported to be associated with impairment of the SWA mechanism in the brain. The reduction of overall SWA, low SWA during NREM sleep, and frequent awakening during sleep have been related to non-restorative, low quality sleep in narcoleptic patients. ${ }^{17,18}$

This study investigated the detailed sleep structures and EEG frequency band abnormalities of narcolepsy through analyzing comparing the PSG data of narcolepsy patients and their age and sex matched controls.

\section{METHODS}

\section{Participants}

We have retrospectively recruited 28 patients first diagnosed with narcolepsy through NPSG and MSLT from July 1st 2010 to June 30th 2015. Individuals with a comorbid psychiatric disease such as depressive disorder, bipolar disorder, ADHD, drug or alcohol abuse; those taking psychiatric drugs (e.g., antidepressants, mood stabilizers, or stimulants); those with thyroid disease; or a neurologic disease (e.g., epilepsy); and those with other sleep disorders (e.g., obstructive sleep apnea, REM sleep behavioral disorder) were excluded. Children and adolescents ( $\leq 19$ years old) were also excluded. Those who disagreed with the use of PSG data or did not submit informed consent were also excluded. Finally, seven narcolepsy patients were included (five males, two females) with a mean age of $26.10 \pm 1.48$ years.

An age and sex matched control group was also recruited from January 1st 2015 to June 30th 2015. As done for the narcolepsy group, individuals with a psychiatric disease, a substance abuse issue, or medical or neurological disease were excluded. Accordingly, the mean age of the seven controls (five males, two females) was $25.90 \pm 1.68$ years. The study protocol was reviewed and approved by the Institutional Review Board of Yeungnam University Hospital. All participants provided informed consent after being informed of the purpose and procedures of this study.

\section{Polysomnography and EEG spectral analysis}

PSG data of the participants were saved in digital form at a sampling rate of $200 \mathrm{~Hz}$. PSG include an EEG to measure brain activity, an electromyography to observe muscle movements (lower jaw and legs), electrooculography to measure the movements of both eyeball, sensors to check air flow through the mouth and nose, breathing efforts in the chest and abdomen, a sensor to measure blood oxygen saturation, and an electro- cardiograph to check heart activity. ${ }^{19}$ In this study, EEG electrodes were attached to the $\mathrm{C} 3 / \mathrm{A} 2, \mathrm{O} 2 / \mathrm{A} 1$ regions according to the international $10-20$ system of electrode placement. ${ }^{20}$ Scoring of the sleep stage was performed by a psychiatrist who majored in sleep medicine according to an internationally authorized sleep scoring system. ${ }^{21}$ For PSG, the CometPLUS PSG system and Twin ${ }^{\circledR}$ PSG software by Grass-Technologies (Astro-Med, Inc., West Warwick, RI, USA) were used.

The C3/A2 brain wave was used for spectral analysis of the sleep EEG. After checking the stage of sleep, all NREM brain activity was analyzed. Signals were conditioned using AS40PLUS amplifiers with low cut filter set at $0.3 \mathrm{~Hz}$ for EEG, 3.0 $\mathrm{Hz}$ for EOG, and $0.03 \mathrm{~Hz}$ for EMG recordings. The EEG epoch with too much artifact was excluded.

We defined an NREM-REM sleep episode to identify the characteristics of brain activity in each sleep cycle. The NREMREM sleep episode was defined from the start of one NREMREM episode to the start of the next, as proposed by Feinberg and Floyd. ${ }^{22}$ In addition, the minimum duration of NREM and REM sleep was respectively defined as 15 minutes and 5 minutes.

We obtained and analyzed EEG data for four sleep cycle from the patients with narcolepsy and controls. Complexity software designed by Laxtha was used to generate brain wave spectra. ${ }^{23}$ Absolute powers (expressed as $\mu \mathrm{V}^{2}$ ) of EEG frequency band were determined using a Fast Fourier Transformation. First, total band power was calculated by adding frequency band powers. Next, the relative power of each band was calculated by dividing band power by total band power. Frequency bands were classified as delta $(0.5-3.9 \mathrm{~Hz})$, theta $(4-7.9 \mathrm{~Hz})$, alpha $(8-13.9 \mathrm{~Hz})$, or beta $(14-30 \mathrm{~Hz})$. Delta and theta activity were compared by NREM sleep cycles between narcolepsy patients and controls.

\section{Statistical analysis}

Data were analyzed using IBM SPSS version 19.0 (IBM Co., Armonk, NY, USA). Statistical significant was defined as $\mathrm{p}<0.05$. The Mann-Whitney $\mathrm{U}$ test was used to compare sex, age, PSG parameters, and relative power changes in NREM sleep cycles. Wilcoxon sign rank test was used to compare relative powers of NREM sleep cycles between two groups.

\section{RESULTS}

\section{Demographical characteristics and sleep parameters}

Age and sex distribution were similar in the narcolepsy and normal control groups. In the comparison of PSG parameters, REM sleep latency in the narcolepsy group (41.00 \pm 19.01 $\mathrm{min}$ ) was significantly shorter than the control group (136.36 $13.62 \mathrm{~min})(\mathrm{p}<0.05)$. Other parameters such as total sleep 
time, sleep efficiency, sleep latency, wake after sleep onset, and distributions of sleep stages were not significantly different between the two groups (Table 1).

\section{EEG spectrum analysis among sleep cycles}

First, we compared the total powers of delta and theta activity of two groups. In comparison of the delta power of the first NREM period, the power in the narcolepsy group ( $0.76 \pm$

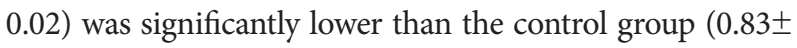
$0.01)(\mathrm{p}<0.05)$. On the other hand, the theta power of the narcolepsy group $(0.15 \pm 0.01)$ was significantly higher than that of the control group $(0.10 \pm 0.01)(\mathrm{p}<0.05)$. For the second NREM sleep period, theta power in the narcolepsy group $(0.18 \pm 0.01)$ was significantly higher than the control group $(0.13 \pm 0.00)(\mathrm{p}<0.05)$. For the third and fourth NREM sleep periods, no difference was seen in the delta and theta power between the two groups. Next, we compared the difference of delta and theta activity by sleep period in each group. In the narcolepsy group, delta wave activities in the second and third NREM periods were significantly lower than those of the first NREM period. Meanwhile, in the control group, delta activities in the second, third, and fourth NREM period were significantly lower than those of the first NREM period ( $\mathrm{p}<0.05)$. In the narcolepsy group, theta activities in the second and third NREM periods were significantly higher than those of the first NREM period. In the control group, theta activities in the second, third, and fourth NREM period were significantly higher than those of the first NREM period $(\mathrm{p}<0.05)$ (Table 2).

\section{DISCUSSION}

In this study, we investigated delta and theta activity differ-

Table 1. Polysomnographic parameters of the study subjects

\begin{tabular}{lccc}
\hline \multirow{2}{*}{ Sleep parameter } & Narcolepsy $(\mathrm{N}=7)$ & Control $(\mathrm{N}=7)$ & $\mathrm{p}$ \\
\cline { 2 - 3 } Total sleep time(min) & Mean \pm SEM & Mean \pm SEM & 0.620 \\
Sleep efficiency (\%) & $393.19 \pm 9.56$ & $402.23 \pm 12.14$ & 0.209 \\
Sleep latency (min) & $87.89 \pm 1.90$ & $92.50 \pm 1.62$ & 0.902 \\
REM sleep latency* (min) & $5.21 \pm 1.69$ & $6.29 \pm 2.64$ & 0.007 \\
Wake after sleep onset (min) & $41.00 \pm 19.01$ & $136.36 \pm 13.62$ & 0.053 \\
Stage 1 (\%) & $49.27 \pm 9.51$ & $26.74 \pm 6.48$ & 0.383 \\
Stage 2 (\%) & $16.44 \pm 3.75$ & $11.46 \pm 2.03$ & 0.318 \\
Stage 3+4 (\%) & $59.95 \pm 3.84$ & $65.03 \pm 2.78$ & - \\
Stage REM (\%) & $7.80 \pm 2.23$ & $9.10 \pm 2.59$ & 0.620 \\
\hline
\end{tabular}

${ }^{*} \mathrm{p}<0.05$. SEM: standard error of the mean, REM: rapid eye movement

Table 2. Comparison of total power of frequencies in narcoleptic patients group over NREM sleep periods

\begin{tabular}{|c|c|c|c|c|c|}
\hline & \multicolumn{2}{|c|}{ Narcolepsy $(\mathrm{N}=7)$} & \multicolumn{2}{|c|}{ Control $(\mathrm{N}=7)$} & \multirow{2}{*}{$\mathrm{p}^{*}$} \\
\hline & Mean $\pm S E M$ & $\mathrm{p}^{\dagger}$ & Mean \pm SEM & $\mathrm{p}^{\dagger}$ & \\
\hline \multicolumn{6}{|c|}{ First NREMP } \\
\hline Delta & $0.76 \pm 0.02$ & & $0.83 \pm 0.01$ & & 0.018 \\
\hline Theta & $0.15 \pm 0.01$ & & $0.10 \pm 0.01$ & & 0.028 \\
\hline \multicolumn{6}{|c|}{ Second NREMP } \\
\hline Delta & $0.68 \pm 0.03$ & 0.018 & $0.76 \pm 0.03$ & 0.018 & 0.063 \\
\hline Theta & $0.18 \pm 0.01$ & 0.028 & $0.13 \pm 0.00$ & 0.018 & 0.018 \\
\hline \multicolumn{6}{|c|}{ Third NREMP } \\
\hline Delta & $0.68 \pm 0.03$ & 0.018 & $0.72 \pm 0.02$ & 0.018 & 0.091 \\
\hline Theta & $0.18 \pm 0.01$ & 0.018 & $0.15 \pm 0.01$ & 0.018 & 0.018 \\
\hline \multicolumn{6}{|c|}{ Fourth NREMP } \\
\hline Delta & $0.68 \pm 0.02$ & 0.128 & $0.70 \pm 0.03$ & 0.018 & 0.735 \\
\hline Theta & $0.17 \pm 0.01$ & 0.176 & $0.16 \pm 0.01$ & 0.018 & 0.310 \\
\hline
\end{tabular}

${ }^{*}$ p value of comparisons between two groups using Wilcoxon Sign Rank Test, ${ }^{\dagger} \mathrm{p}$ value of comparisons between the first NREMP and the second, third and fourth NREMP. NREMP: non-rapid eye movement period, SEM: standard error of the mean, Delta $(0.5-3.9 \mathrm{~Hz})$, Theta $(4-7.9 \mathrm{~Hz})$ 
ences between narcolepsy patients and normal controls using EEG spectrum analysis. The major finding of this study is that while the decrease in delta activity in the control group lasted from the first to fourth NREM episodes, the decrease only occurred from the first to third NREM episodes in narcolepsy group. There have been similar results in previous studies. In a study of 11 drug-naïve patients with narcolepsy-cataplexy and 11 matched controls, Khatami et al. ${ }^{18}$ reported that progressive decay of the power density of the $0.75-3.75 \mathrm{~Hz}$ band in narcolepsy group did not occur.

Guilleminault et al. ${ }^{17}$ also reported that delta activity continuously reduced from the beginning to the end of sleep in a control group but not in a narcolepsy group. It can be assumed that narcolepsy patients are likely to have a certain kind of delta activity related sleep-disturbance mechanism in NREM sleep. ${ }^{15,17}$

In this study, there was a difference in NREM theta activity between the two groups. While the increase in theta activity in the control group lasted from the first to fourth NREM episodes, the increase only occurred from the first to third NREM episodes in the narcolepsy group. There have not been sufficient studies regarding the difference in theta activity of narcolepsy patients and controls so far. A study conducted by eight drug-naïve narcoleptic patients and age-matched normal volunteers reported that while the control group showed a significant theta band increase through the sleep cycle, narcoleptic patients did not show this increase. ${ }^{15}$ The precise mechanism of the theta band change through the sleep cycle is not yet known. It is possible that high amplitude theta wave burst might relate to Hcrt deficiency, one of the major pathophysiology of narcolepsy. ${ }^{24}$ Additional studies are necessary to elucidate this.

The next finding of this study was that narcolepsy patients showed reduced REM sleep latency. This finding is also consistent with other studies. Vernet and Arnulf reported that REM sleep latency was reduced in a narcolepsy group regardless of sleep time. ${ }^{25}$ Mukai et al. ${ }^{15}$ also reported a significant reduction in REM sleep latency in narcoleptic patients despite no differences in total sleep time, sleep efficiency, and stage REM sleep. Short REM latency is a frequent and specific finding in narcolepsy, and $30-40 \%$ of patients have SOREMP as identified by nocturnal PSG. ${ }^{26}$

This study has some limitations. First, the sample size was small, and thus, we could not subdivide narcolepsy patients into two types (narcolepsy with cataplexy and without cataplexy). For this reason, the generalizability of the results is limited. Further, more detailed studies, with larger narcolepsy patient groups, are required in the future. Second, there was a difference between the period when NPSG was conducted with patients and the recruited control group. While the NPSG data of narcolepsy patients were retrospectively collected from July 1st 2010 to June 30th 2015, the NPSGs of the control group were conducted from January 1st to June 30th in 2015. This may lead to a limitation of the interpretations of the results of the study because of the different time periods. Regardless of these limitations, this study suggested the possibility that the change in NREM delta and theta activity during nocturnal sleep of narcolepsy patients differed from the control group, and these differences might be related to pathophysiology of narcolepsy.

\section{Acknowledgments}

This research was supported by a grant of Yeungnam University Medical Center (2010).

\section{REFERENCES}

1. Bassetti C, Aldrich MS. Narcolepsy. Neurol Clin 1996;14:545-571.

2. Zorick F, Roehrs T, Wittig R, Lamphere J, Sicklesteel J, Roth T. Sleepwake abnormalities in narcolepsy. Sleep 1986;9:189-193.

3. American Academy of Sleep Medicine. The International Classification of Sleep Disorders: Diagnostic and Coding Manual, ICSD-2. Westchester, IL: American Academy of Sleep Medicine; 2005.

4. Kim J. Linear/non-linear tools and their applications to sleep eeg: spectral, detrended fluctuation, and synchrony analyses. Sleep Med Psychophysiol 2008;15:5-17.

5. Frauscher B, Gschliesser V, Brandauer E, Schonwald SV, Falkenstetter T, Ehrmann L, et al. Motor disturbances during non-REM and REM sleep in narcolepsy-cataplexy: a video-polysomnographic analysis. J Sleep Res 2011;20:514-521.

6. Montplaisir J, Billiard M, Takahashi S, Bell IR, Guilleminault C, Dement WC. Twenty-four-hour recording in REM-narcoleptics with special reference to nocturnal sleep disruption. Biol Psychiatry 1978;13:73-89.

7. Montplaisir J, Godbout R. Nocturnal sleep of narcoleptic patients: revisited. Sleep 1986;9:159-161.

8. Broughton R, Dunham W, Newman J, Lutley K, Duschesne P, Rivers M. Ambulatory 24 hour sleep-wake monitoring in narcolepsy-cataplexy compared to matched controls. Electroencephalogr Clin Neurophysiol 1988;70:473-481.

9. Mattarozzi K, Bellucci C, Campi C, Cipolli C, Ferri R, Franceschini C, et al. Clinical, behavioural and polysomnographic correlates of cataplexy in patients with narcolepsy/cataplexy. Sleep Med 2008;9:425-433.

10. Black J, Pardi D, Hornfeldt CS, Inhaber N. The nightly use of sodium oxybate is associated with a reduction in nocturnal sleep disruption: a double-blind, placebo-controlled study in patients with narcolepsy. J Clin Sleep Med 2010;6:596-602.

11. Campbell AJ, Signal TL, O’Keeffe KM, Bakker JP. Narcolepsy in New Zealand: pathway to diagnosis and effect on quality of life. N Z Med J 2011;124:51-61.

12. Baier PC, Hallschmid M, Seeck-Hirschner M, Weinhold SL, Burkert S, Diessner N, et al. Effects of intranasal hypocretin-1 (orexin A) on sleep in narcolepsy with cataplexy. Sleep Med 2011;12:941-946.

13. Kim JW, Shin HB, Robinson PA. Quantitative study of the sleep onset period via detrended fluctuation analysis: normal vs. narcoleptic subjects. Clin Neurophysiol 2009;120:1245-1251.

14. Jenkins W, McMillan JW. Activation analysis with standards containing two or more active nuclides A computerized method of calculation involving decay and gamma spectral resolution. Talanta 1968;15:11651175 .

15. Mukai J, Uchida S, Miyazaki S, Nishihara K, Honda Y. Spectral analysis of all-night human sleep EEG in narcoleptic patients and normal subjects. J Sleep Res 2003;12:63-71. 
16. Borbely AA. A two process model of sleep regulation. Hum Neurobiol 1982;1:195-204.

17. Guilleminault C, Heinzer R, Mignot E, Black J. Investigations into the neurologic basis of narcolepsy. Neurology 1998;50(2 Suppl 1):S8-S15.

18. Khatami R, Landolt HP, Achermann P, Retey JV, Werth E, Mathis J, et al. Insufficient non-REM sleep intensity in narcolepsy-cataplexy. Sleep 2007;30:980-989.

19. James G, Paul C, Troy P. Atlas of Polysomnography. Second Ed. Philadelphia: Lippincott Williams \& Wilkins; 2010.

20. Jasper $\mathrm{H}$. The 10-20 electrode system of the International Federation. Electroencephalogr Clin Neurophysiol 1958;10:370-375.

21. Iber C, Ancoli-Israel S, Chesson AL Jr, Quan SF; for the American Academy of Sleep Medicine. The AASM Manual for the Scoring of Sleep and
Associated Events: Rules, Terminology and Technical Specifications. 1st Ed. Westchester, IL: American Academy of Sleep Medicine; 2007.

22. Feinberg I, Floyd TC. Systematic trends across the night in human sleep cycles. Psychophysiology 1979;16:283-291.

23. Laxtha. Complexity User manual. Dae-Jeon: Laxtha Inc; 2004.

24. Lo Martire VC, Bastianini S, Berteotti C, Silvani A, Zoccoli G. High amplitude theta wave bursts: a novel electroencephalographic feature of rem sleep and cataplexy. Arch Ital Biol 2015;153:77-86.

25. Vernet C, Arnulf I. Narcolepsy with long sleep time: a specific entity? Sleep 2009;32:1229-1235.

26. Aldrich MS, Chervin RD, Malow BA. Value of the multiple sleep latency test (MSLT) for the diagnosis of narcolepsy. Sleep 1997;20:620-629. 